\title{
ARTICLE
}

\section{Spatiotemporal isolation of attosecond soft X-ray pulses in the water window}

Francisco Silva ${ }^{1, \star}$, Stephan M. Teichmann ${ }^{1, \star}$, Seth L. Cousin ${ }^{1, \star}$, Michael Hemmer $^{1}$ \& Jens Biegert ${ }^{1,2}$

Attosecond pulses at photon energies that cover the principal absorption edges of the building blocks of materials are a prerequisite for time-resolved probing of the triggering events leading to electronic dynamics such as exciton formation and annihilation. We demonstrate experimentally the isolation of individual attosecond pulses at the carbon K-shell edge $(284 \mathrm{eV})$ in the soft X-ray water window with pulse duration below 400 as and with a bandwidth supporting a 30-as pulse duration. Our approach is based on spatiotemporal isolation of long-wavelength-driven harmonics and validates a straightforward and scalable approach for robust and reproducible attosecond pulse isolation.

\footnotetext{
${ }^{1}$ ICFO-Institut de Ciencies Fotoniques, Mediterranean Technology Park, 08860 Castelldefels (Barcelona), Spain. ${ }^{2}$ ICREA-Institució Catalana de Recerca i Estudis Avançats, 08010 Barcelona, Spain. ${ }^{*}$ These authors contributed equally to this work. Correspondence and requests for materials should be addressed to J.B. (email: jens.biegert@icfo.eu).
} 
T he power of attosecond science lies in its capability for time-resolving electronic motion on its native ultrafast timescale $^{1,2}$, that is, observation of the previously intractable 'triggering events' leading to electronic excitation, formation of charge density waves, changes of chemical bonds or the emergence of ultrafast magnetism, just to mention a few examples. The fundamental requirements for such investigations are isolated attosecond-duration pulses in synchronicity with excitation or interrogation pulses and at photon energies that permit probing the relevant mechanisms. For instance, the low efficiency of organic solar cells is related to a lack of knowledge of the ultrafast events within the energy transformation processes, that is, ultrafast exciton formation and annihilation. Modern organic solar cells consist of metal ligand hydrocarbon complexes and attosecond-duration pulses with photon energy of $284 \mathrm{eV}$, as demonstrated here, will permit probing exciton dynamics at the $\mathrm{K}$-shell of the carbon atom of the solar cell.

Isolated attosecond pulses are generated by confining high harmonic generation (HHG) driven with an intense laser to a short temporal window that is on the order of the field cycle duration of the driving laser. This condition can be met by either producing few-cycle duration pulses ${ }^{3}$ or through a variety of methods that effectively confine the recollision to a similar time $\operatorname{span}^{4-6}$. In order to generate repeatable attosecond pulses and to prevent random timeshifts, which would range on the order of the field cycle duration, control over the generating laser's electric carrier-to-envelope (CEP) field waveform is required. The maximum reachable photon energy from HHG, the so-called cutoff, can be determined with the classical three-step model of recollision ${ }^{7}$, which is dependent on the harmonic generation medium, the laser peak intensity and scales with the square of the laser wavelength. The demanding requirements for single-attosecond pulse generation have so far only been met with advanced Ti:Sapphire laser sources at $800 \mathrm{~nm}$, which have confined attosecond generation largely to photon energies below $150 \mathrm{eV}$. Ponderomotive scaling of HHG to $\mathrm{keV}$ photon energies has been demonstrated by increasing the Ti:Sapphire laser's peak intensity ${ }^{8}$; however, the attainable non-phase-matched harmonic yield is low and further intensity scaling is limited by depletion of the medium's ground state. Exploiting wavelength scaling is therefore the route most actively pursued by many groups in the field, with a $\mathrm{keV}$ cutoff being demonstrated when driven by a mid-infrared source ${ }^{9}$. This has resulted in an upsurge in the development of long-wavelength ultrafast light sources on the basis of parametric processes. One standing issue for HHG was the unfavourable scaling of the single-atom response with laser wavelength ${ }^{10-13}$. This was shown, however, to be largely mitigable by employing high-pressure phase matching ${ }^{14}$, which results in appreciable photon yields ${ }^{15-17}$. Recently, our group demonstrated the first soft X-ray absorption fine structure measurement at the carbon K-edge from a solid material ${ }^{17}$, thereby validating the feasibility of this approach for real applications in solid-state physics.

In order to produce isolated attosecond pulses at high photon energies, high-pressure phase-matching of long-wavelengthdriven $\mathrm{HHG}$ may employ ionization gating 5,18 , limiting emission to a single laser cycle $^{19}$. Nevertheless, to be useful for time-resolved measurements, an additional requirement is driving attosecond generation with a CEP-controlled source since the absence of CEP stability would result in random timing slips of the attosecond emission on the order of the half cycle duration of the driving field; a 2,000-nm driver wavelength corresponds to 3,000 as for the duration of half a cycle. Recently, the first CEP-dependent HHG spectra were demonstrated at $300 \mathrm{eV}$ (ref. 15) and at $400 \mathrm{eV}$ (ref. 17).
Here we address the to date unsolved problem of isolation of a single-attosecond pulse in a photon energy range above $150 \mathrm{eV}$. We demonstrate experimentally at the carbon K-edge at $284 \mathrm{eV}$ spatiotemporal isolation (STI) of a single-attosecond pulse in the water window.

\section{Results}

Implementation of wavefront rotation for soft X-ray emission at the carbon K-edge. Our laser system generates pulses at $1,850-\mathrm{nm}$ wavelength at a repetition rate of $1 \mathrm{kHz}$ with a pulse duration of $13 \mathrm{fs}$. These pulses carry an energy of $230 \mu \mathrm{J}$ and are CEP-stable to $80 \mathrm{mrad}$ r.m.s. over $1 \mathrm{~h}$ (ref. 17,20). We use a 100-mm-focal length spherical mirror to focus the pulses into the HHG target, which consists of a $1.5-\mathrm{mm}$-outer diameter tube with $0.3-\mathrm{mm}$ entrance and exit holes through which the beam is focused; see the Methods section for further details and Fig. 1 for a schematic.

The laser radiation is filtered out by transmitting the harmonic beam through a $100-\mathrm{nm}$ free-standing aluminium filter; hereafter, the harmonics are refocused with a grazing-incidence ellipsoidal mirror into a home-built soft X-ray spectrograph for analysis. The spectrograph consists of an aberration-corrected 2,400-1 $\mathrm{mm}^{-1}$-flat-field grating (Hitachi) and a cooled backilluminated soft X-ray CCD (charge-coupled device) camera (PIXIS-XO-2048B, Princeton Instruments). For STI of the attosecond emission, we employ a wavefront rotation scheme in the generation medium, also termed attosecond lighthouse $\mathrm{s}^{21,22}$ or photonic streaking ${ }^{18}$. Wavefront rotation at $1.3 \mathrm{mrad} \mathrm{fs}^{-1}$ is implemented by inserting a thin (uncoated) fused silica wedge with a 5-degree apex angle before the focusing mirror. The maximum reachable peak intensity on the target is $0.35 \mathrm{PW} \mathrm{cm}^{-2}$, which is reduced to $0.3 \mathrm{PW} \mathrm{cm}^{-2}$ when inserting the uncoated wedge. We note that even greater beamlet separation than that demonstrated is possible but on expense of peak intensity on target, hence requiring higher laser pulse energy than available in our experiment. The wavefront rotation of the generating field translates directly into wavefront rotation of the generated harmonic field, leading to a directional change of the emission. Figure 1 illustrates the effect with a measured spatiospectral and a simulated spatiotemporal distribution of the generating pulse, as well as a far-field profile of the generated HHG radiation. While the experimental conditions were optimized for a water window cutoff with neon, we choose to first illustrate the effect of wavefront rotation with argon in Fig. $1 c, f$ as it results in emission over a larger spectral range at lower photon energy. Nicely visible is the directional change of the entire harmonic emission and the associated change in spectral energy distribution without (Fig. 1c) and with wavefront rotation (Fig. 1f).

Photonic streaking of $300-\mathrm{eV}$ soft $\mathrm{X}$-ray emission. To experimentally demonstrate our STI approach for isolation of a single-attosecond pulse in the water window, we use neon as a generation medium as it facilitates HHG at the carbon K-edge at $284 \mathrm{eV}$ with the pulse intensities available from our set-up and for 3.6 bar backing pressure; the pressure dependence was recently demonstrated in ref. 17. The short pulse duration, and consequentially strong dependence on the CEP, permits an unambiguous investigation of the feasibility of isolating attosecond structures at this unprecedentedly high photon energy range using photonic streaking as each successive attosecond burst is launched in a different direction (here called beamlet), and thus is readily distinguishable. The measurement reported in Fig. 2 performed with WFR shows that, as the CEP is changed, we observe a continuous movement of a single beamlet 

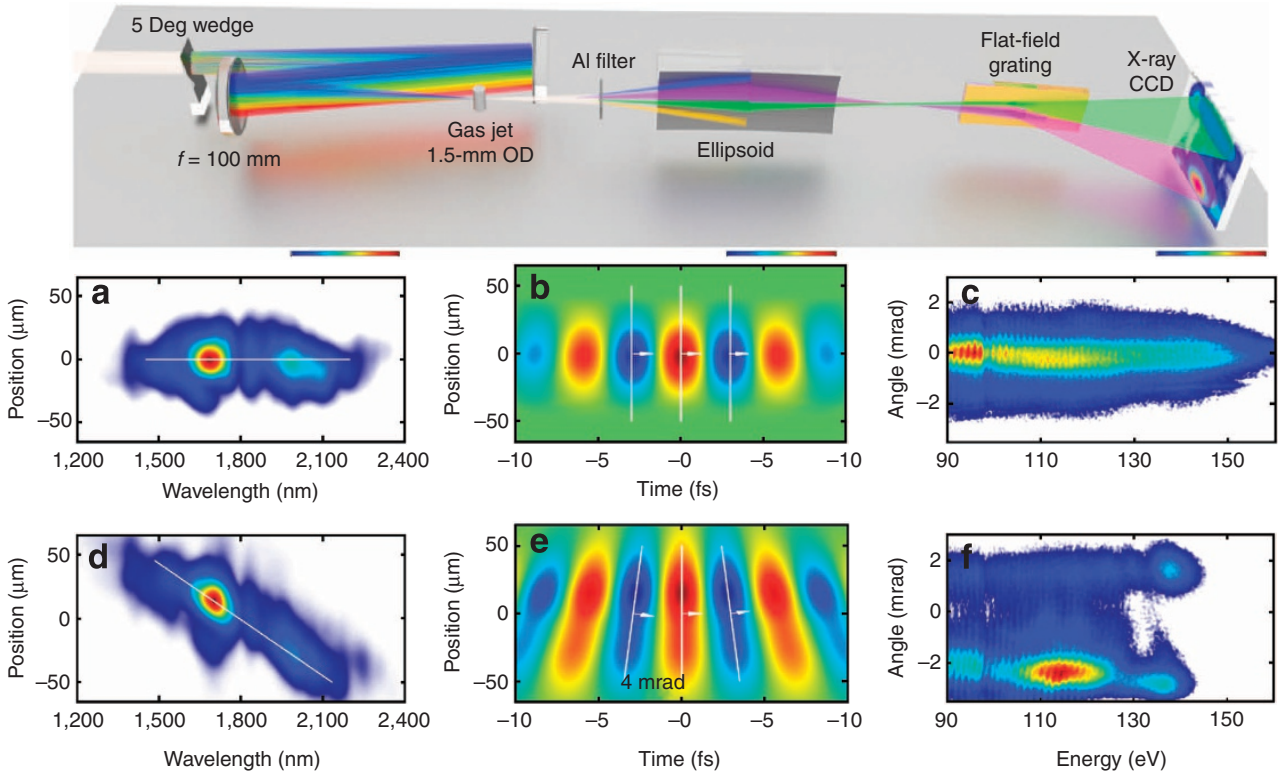

Figure 1 | Experimental set-up and conditions. Top: schematic of the experimental set-up. (a) Spatiospectral profile in the gas jet focus without WFR. (b) Corresponding calculated electric field. (c) Measured HHG spatiospectral profile generated in 1-bar Argon without WFR. (d) Spatiospectral profile in the gas jet focus with WFR. (e) Corresponding calculated electric field. (f) Measured HHG spatiospectral profile generated in 1-bar Argon with WFR, revealing a 4-mrad separation between beamlets.
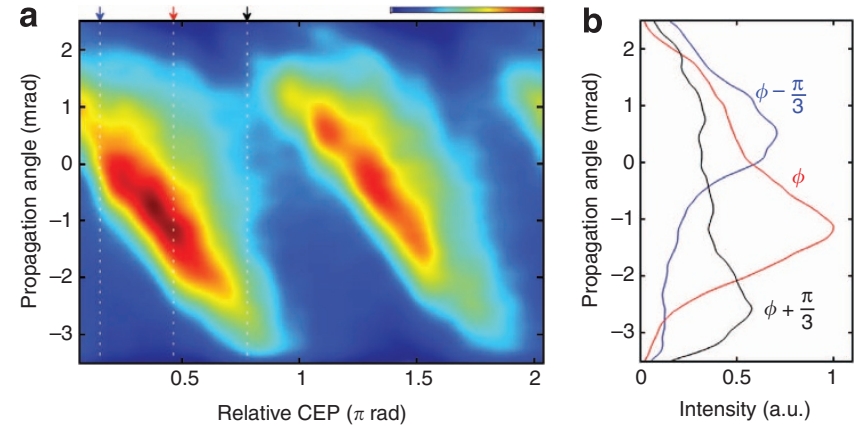

Figure 2 | CEP control of emission angle. (a) Spatial profile of the emitted radiation in function of CEP, revealing a clear control of the emission angle. (b) Spatial profile for three CEPs. The spectra are integrated from 200 to $400 \mathrm{eV}$.

with fairly constant angular extent over the entire CEP range. The clear dependence of the angular drift with CEP, and the clean angular distribution, is already an indication of emission of an attosecond pulse structure ${ }^{18}$ since even a small pre- or post-pulse content (on the order of a few percent) would manifest itself in modulation of the angular content. see Fig. $2 \mathrm{~b}$ for a comparison between integrated spectra for three different CEP values.

STI of a single-attosecond pulse in the water window. We investigate the angular separation of beamlets and therefore our ability to steer and isolate single-attosecond structures in the water window. Inserting the wedge reduces the peak intensity on target by $15 \%$, but still results in a $300 \mathrm{eV}$ cutoff; this value is also inferred from the classical cutoff law ${ }^{23}$. Figure 3 shows the result of the measurement for three different relative CEP values of $\Phi$, $\Phi+\pi / 3$ and $\Phi+2 \pi / 3$, with the spectrograph imaging the focal plane of the ellipsoidal mirror along the energy axis. Shown to the right is the emerging spectrum for angular integration over $2.5 \mathrm{mrad}$ and for each case.
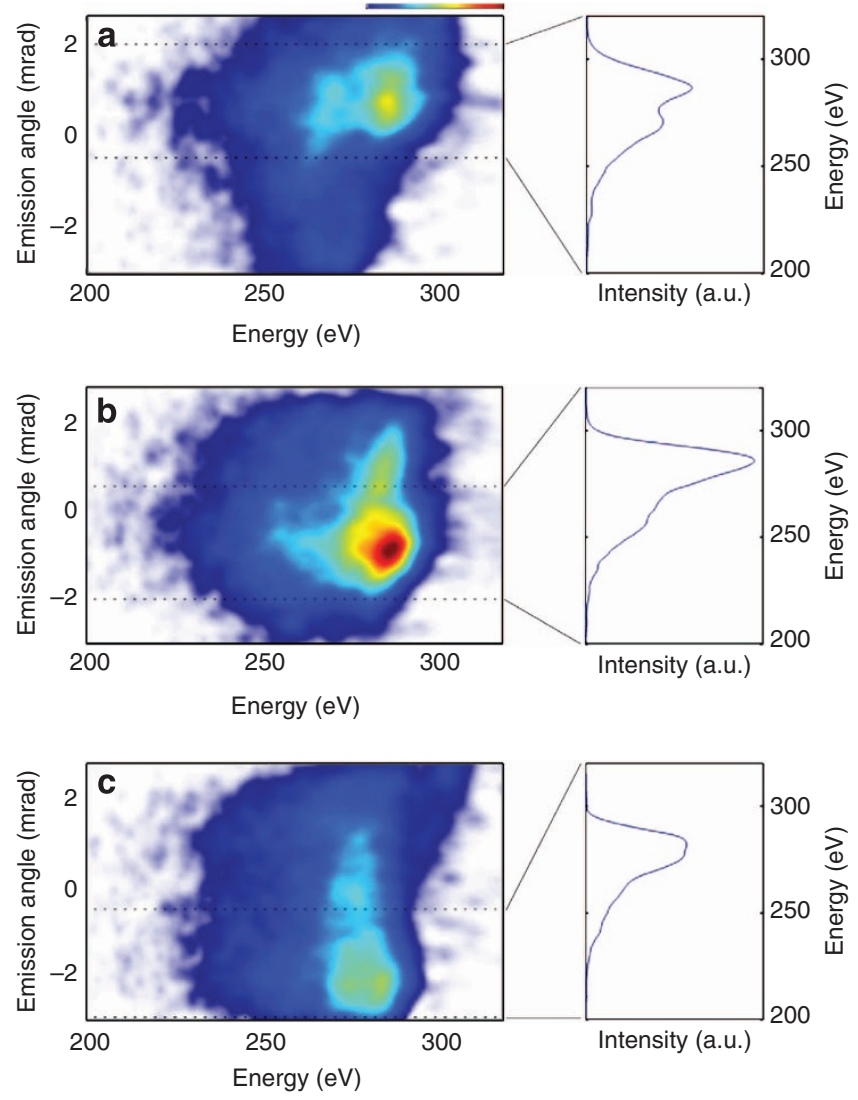

Figure 3 | Isolated spatiotemporal profiles for three different emission angles $(\mathbf{a}-\mathbf{c})$. Shown is the result of the measurement for three different relative CEP values of $\Phi, \Phi+\pi / 3$ and $\Phi+2 \pi / 3$ with the spectrograph imaging the focal plane of the ellipsoidal mirror along the energy axis. The dotted lines indicate the 2.5-mrad-integration region for the spectra on the right. 
Figure 3a exhibits a fairly broad spectrum supporting a 59-asduration pulse. Next, we increased the CEP by $\pi / 3$, which results in a broad and structureless spectral emission shown in Fig. $3 \mathrm{~b}$ and thus supports the shortest 57-as-duration water window pulse. Further incrementing CEP to $2 \pi / 3$, we observe in Fig. $3 \mathrm{c}$ a $10 \mathrm{eV}$ downshift of the highest emitted photon energy to $290 \mathrm{eV}$, whereas the overall spectral shape remains. The persistence of the spectra for the isolated emission and the angular emission dependence on CEP is the first experimental evidence of isolated attosecond pulse generation in the water window at the carbon K-edge. On the basis of this clear demonstration of STI and the generation of single-attosecond structures, we turn to a numerical code (described in Methods) to simulate the wavefront rotation effect in order to gain further insight into the interplay of the various quantum trajectories and the effectiveness of STI.

Shown in Fig. 4a is the spatiotemporal profile of the generating pulse and the effect of wavefront rotation where the harmonics are generated. The simulated far-field angular-spectral profile is depicted in Fig. 4b, and it shows the expected interferences due to emission from the different trajectories (long and short). The corresponding spectrogram is shown in Fig. 4c. Our measurements (Fig. 3) do not exhibit signatures of such trajectory interferences and are in accordance with the findings in ref. 18, which demonstrated both experimentally and with twodimensional simulations that only the short trajectories add up coherently to the macroscopic signal. Thus, we numerically removed the long trajectory contribution in our single-atom calculations; the results are shown in the bottom row of Fig. 4 . The far-field pattern of Fig. 4e shows qualitative agreement with the measurement in Fig. 3. Figure 4 b,e shows how half cycle emissions are streaked on the CCD camera. We find that the most prominent half cycle at $-1 \mathrm{fs}$ is emitted on axis, and is registered at the camera around $0 \mathrm{mrad}$. The spectrogram of the resulting soft X-ray emission is shown in Fig. $4 \mathrm{f}$ and demonstrates prominent emission at the half cycle cutoff. The next, less prominent, half cycle emission at $2 \mathrm{fs}$ is slightly upshifted and detected at 3 mrad. For completeness, the case for only the long trajectories is shown in Supplementary Figs 1-3; however, it does not show the same level of agreement with the measurement as shown in Fig. 4e. For comparison, we also show harmonic emission for the case without wavefront rotation over the identical CEP range (Fig. 5). Finally, we find that the simple single-atom model qualitatively matches with our experimental investigations and evidences a predominant contribution from the short trajectory. The strong dependence of the cutoff and angular emission on CEP, as well as the clean isolation of singleattosecond bursts, are first experimental evidence of isolated attosecond pulse generation in the water window at the carbon K-edge of $284 \mathrm{eV}$ and show the applicability of STI to unprecedentedly high photon energies.

Efficiency of STI. Next, we establish the efficiency of the STI method for water window radiation and its influence on photon yield and pre- and post-pulse contrast. For practical purposes, we contrast the implemented attosecond pulse isolation scheme against generation with a few-cycle pulse without wavefront rotation (see Fig. 5).

We find that STI leads to a fivefold decrease in peak photon yield (blue curve Fig. 5a) compared with the implementation without STI (black curve). For a comparison of the flux for the attosecond emission, however, the case is favourable for STI. In order to compare the two cases, we rely on our simulations. With STI, an iris can simply be inserted into the soft X-ray beam to isolate a single-attosecond pulse structure; the resulting measured
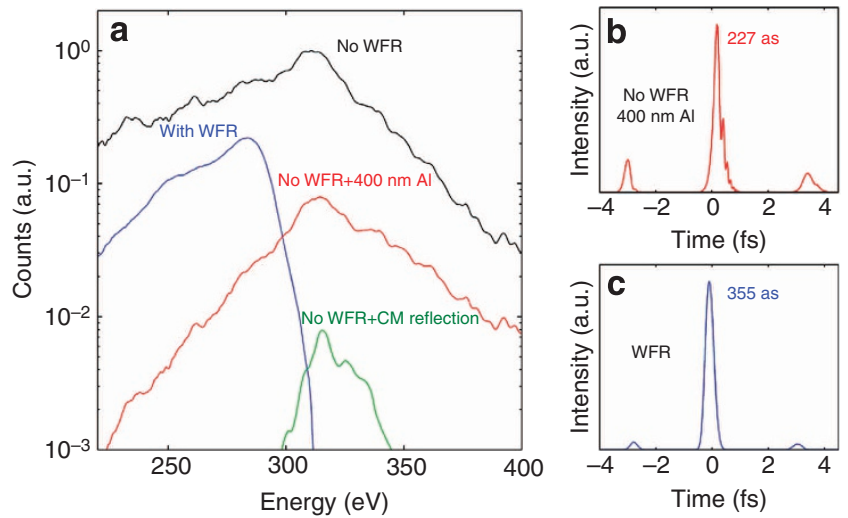

Figure 5 | Photon yield and calculated pulse profiles. (a) Comparison of photon yields on target. Chirped mirror reflection profile from Ref. 24 (b). Simulated pulse profile in the case without wavefront rotation, after transmission through a 400-nm Al filter, leading to a pre/post-pulse contrast of 1:5. (c) Simulated pulse profile in the case of wavefront rotation, leading to an increased pre/post-pulse contrast of 1:20.
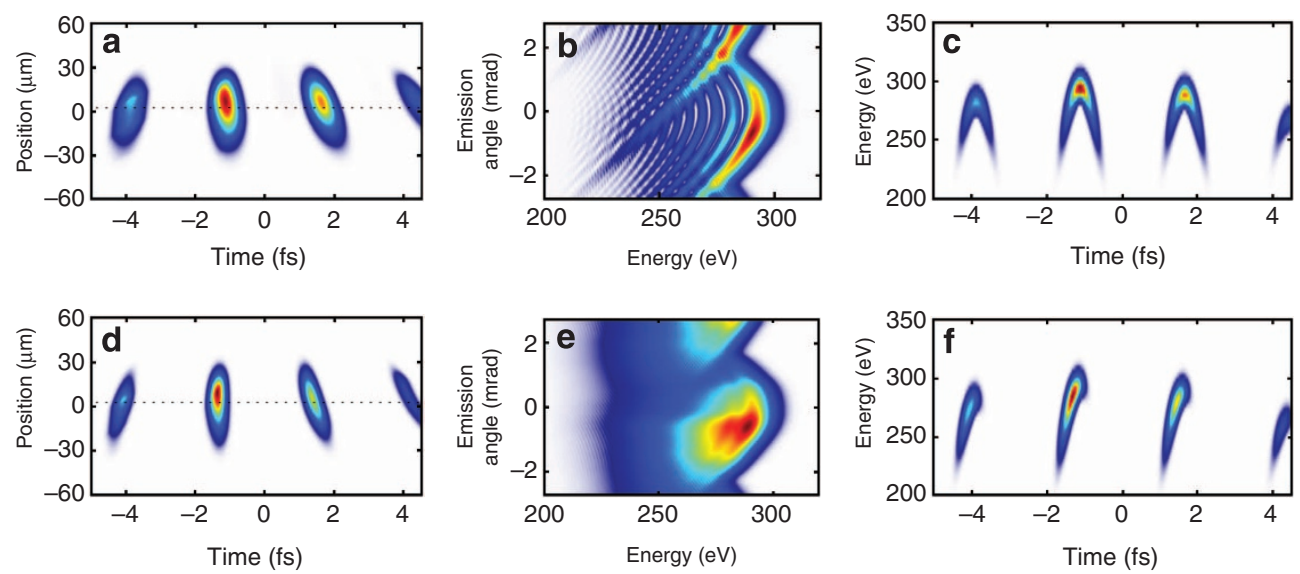

Figure 4 | Single-atom response WFR simulation results. Shown in the top row (a-c) are results including short and long trajectories, whereas the bottom row (d-f) shows results for identical simulation values, but with the long trajectory numerically removed. Panels a,d show the generated spatiotemporal profile in focus; $(\mathbf{b}, \mathbf{e})$ angular-spectral profile in the far field, for an angular range of $5.5 \mathrm{mrad}$; (c,f) spectrogram of the pulse, calculated for the electric field along the dotted line in $\mathbf{a}$. The case for long trajectories is only displayed in the Supplementary Information for completeness. 
photon yield is shown for the blue curve in Fig. 5a. Without STI, the $100-\mathrm{nm}$ Al filter will not sufficiently suppress emission from pre- and post-half cycles, thereby resulting in multiple attosecond pulse emission. Increasing the thickness of the soft X-ray filter to $400 \mathrm{~nm} \mathrm{Al}$ achieves a pre/post-pulse contrast of 1:5 and calculated soft X-ray pulse duration of 230 as (Fig. 5b). In contrast, STI results in a superior 1:20 contrast but a longer calculated 355-as duration (Fig. 5d). For comparison, we also show the performance of a state-of-the-art multilayer mirror ${ }^{24}$ for the water window (green curve in Fig. 5a). By contrasting the relative efficiency of all schemes for generating a single-attosecond pulse, we find that the STI scheme presents a very attractive solution, resulting in a higher photon energy and better pre/post-pulse contrast than without WFR, as in that case the metallic filters or dielectric reflectors required to select a single-attosecond pulse lead to a high penalty in photon flux. A higher pulse contrast is also predicted with the STI scheme, as the attosecond pulse can be selected spatially rather than spectrally.

\section{Discussion}

We have demonstrated the isolation of a single-attosecond pulse at the carbon K-shell edge at $284 \mathrm{eV}$ in the water window using STI on the basis of wavefront rotation. Our results validate the approach as a viable route for the isolation of a single-attosecond pulse in a soft X-ray energy regime in which the commonly used spectral filtering scheme with metal foils remains to be demonstrated, or whenever tunability of the emission energy may be required. In addition, tuning emission to two attosecond bursts, which are emitted into two different angular directions presents a route for the implementation of efficient soft X-ray pump/soft X-ray probe schemes in the high photon energy range by spatial recombination of two successive attosecond bursts and their relative pump probe delay via independent refocusing optics. Another attractive aspect of the spatial isolation method is the marginal penalty in photon flux, which, when compared with spectral filtering in the same energy regime, is even energetically superior. Our experimental demonstration is the first proof of the feasibility of implementation of such a single-attosecond pulseselection regime for ponderomotively scaled sources, which can reach the water window spectral regime and may become a major asset for the evolution of attosecond science into the energy range important for material science and biology.

\section{Methods \\ Experiment. High-energy pulses from a Ti:Sapphire amplifier system with $40 \mathrm{fs}$ duration and $7 \mathrm{~mJ}$ energy at $1 \mathrm{kHz}$ repetition rate are frequency-converted to $1.85 \mu \mathrm{m}$ using an optical parametric amplifier, resulting in CEP-stable $0.8 \mathrm{~mJ}$ pulses with $40 \mathrm{fs}$ duration. These pulses are spectrally broadened through nonlinear propagation in a hollow core fibre filled with 1.5 bar of argon, which both broadens the spectrum and introduces adequate dispersion for subsequent compression down to $13 \mathrm{fs}$ with bulk material ${ }^{17,20}$. The CEP-stable pulses are then focused with an $f=100$-mm spherical mirror into a gas target $(1.5 \mathrm{~mm}$ outer diameter and $0.5 \mathrm{~mm}$ inner diameter) with $0.3 \mathrm{~mm}$ diameter entrance and exit holes, filled with neon at a backing pressure of 3.6 bar. The residual fundamental radiation is filtered with a $100-\mathrm{nm}$ free-standing aluminium foil and the transmitted radiation is refocused using a grazing-incidence ellipsoidal mirror (Zeiss). A home-built spectrograph, consisting of a motorized slit, flat-field imaging reflective grating (Hitachi, 2,400 lines per $\mathrm{mm}$ ) and a cooled, back-illuminated CCD (Princeton Instruments) is used to measure the spatiospectral profile of the radiation in function of CEP. Photon counts are extracted using the measured spectra, the camera quantum efficiency and the measured grating diffraction efficiency.}

Simulations. The simulation results presented in the Discussion section were obtained with a model based on two different HHG codes, one for the threedimensional propagation without WFR and the other for the microscopic response with WFR. In the case of no WFR, cylindrical symmetry is assumed and nonlinear propagation using pseudospectral methods is used to numerically propagate the driving laser electric field. Dispersion, diffraction, self-phase modulation, plasma dephasing and absorption are taken into account ${ }^{25}$. Ionization rates are calculated using the Ammosov-Delone-Krainov formula ${ }^{26}$. After calculation of the driving laser field the single-atom HHG response is computed at every point of the propagation grid (resampled to provide appropriate resolution) using an augmented Lewenstein model ${ }^{13,27}$ with an electron birth factor that incorporates the Ammosov-Delone-Krainov ionization rate $e^{28-30}$ and photorecombination cross-section $^{31}$ for the recombination step ${ }^{32}$. Gaussian input pulses in the spatial and temporal domains were considered, and the target was considered to have a density of $1.2 \times 10^{19}$ atoms per $\mathrm{cm}^{3}$. The simulated target thickness was the full inner diameter $(0.5 \mathrm{~mm})$. This simulation was used to calculate the results in Fig. 5b. In the case of WFR, the single-atom response was calculated across the spatial dimension of the beam, using the code described above ${ }^{33,34}$. The WFR was introduced by modelling the electric field using the measured quantities in Fig. 1d,e, and using Gaussian integrated spectral and spatial profiles to minimize numerical noise. This simulation was used to calculate the results of Figs 4 and $5 c$ The pulse is propagated into the far field through Fourier analysis, in order to calculate the spatiospectral profile of Fig. $4 \mathrm{~b}$,e.

\section{References}

1. Schultze, M. et al. Controlling dielectrics with the electric field of light. Nature 493, 75-78 (2013).

2. Schultze, M. et al. Delay in photoemission. Science 328, 1658-1662 (2010).

3. Goulielmakis, E. et al. Single-cycle nonlinear optics. Science 320, 1614-1617 (2008).

4. Mashiko, H. et al. Double optical gating of high-order harmonic generation with carrier-envelope phase stabilized lasers. Phys. Rev. Lett. 100, 103906 (2008).

5. Ferrari, F. et al. High-energy isolated attosecond pulses generated by abovesaturation few-cycle fields. Nat. Photon. 4, 875-879 (2010).

6. Sansone, G. et al. Isolated single-cycle attosecond pulses. Science 314, 443-446 (2006).

7. Corkum, P. B. Plasma perspective on strong field multiphoton ionization. Phys Rev. Lett. 71, 1994 (1993).

8. Spielmann, C. et al. Generation of coherent X-rays in the water window using 5-femtosecond laser pulses. Science 278, 661-664 (1997).

9. Popmintchev, T. et al. Bright coherent ultrahigh harmonics in the keV X-ray regime from mid-infrared femtosecond lasers. Science 336, 1287-1291 (2012).

10. Tate, J. et al. Scaling of wave-packet dynamics in an intense mid-infrared field. Phys. Rev. Lett. 98, 13901 (2007).

11. Shiner, A. et al. Wavelength scaling of high harmonic generation efficiency. Phys. Rev. Lett. 103, 73902 (2009).

12. Frolov, M. V. et al. Analytic description of the high-energy plateau in harmonic generation by atoms: can the harmonic power increase with increasing laser wavelengths? Phys. Rev. Lett. 102, 243901 (2009).

13. Austin, D. R. \& Biegert, J. Strong-field approximation for the wavelength scaling of high-harmonic generation. Phys. Rev. A 86, 023813 (2012).

14. Popmintchev, T. et al. Phase matching of high harmonic generation in the soft and hard X-ray regions of the spectrum. Proc. Natl Acad. Sci. USA 106, 10516-10521 (2009).

15. Ishii, N. et al. Carrier-envelope phase-dependent high harmonic generation in the water window using few-cycle infrared pulses. Nat. Commun. 5, 3331 (2014).

16. Ding, C. et al. High flux coherent super-continuum soft X-ray source driven by a single-stage, $10 \mathrm{~mJ}$, Ti:sapphire amplifier-pumped OPA. Opt. Exp. 22, 6194-6202 (2014).

17. Cousin, S. et al. High-flux table-top soft X-ray source driven by sub-2-cycle, CEP stable, $1.85 \mu \mathrm{m} \mathrm{1-kHz}$ pulses for carbon K-edge spectroscopy. Opt. Lett. 39, 5383-5386 (2014).

18. Kim, K. T. et al. Photonic streaking of attosecond pulse trains. Nat. Photon. 7, 651-656 (2013).

19. Chen, M.-C. et al. Generation of bright isolated attosecond soft X-ray pulses driven by multicycle mid infrared lasers. Proc. Natl Acad. Sci. USA 111, E2361-E2367 (2014).

20. Schmidt, B. E. et al. Compression of $1.8 \mu \mathrm{m}$ laser pulses to sub two optical cycles with bulk material. Appl. Phys. Lett. 96, 121109 (2010).

21. Vincenti, H. \& Quéré, F. Attosecond lighthouses: how to use spatiotemporally coupled light fields to generate isolated attosecond pulses. Phys. Rev. Lett. 108, 113904 (2014).

22. Wheeler, J. A. et al. Attosecond lighthouses from plasma mirrors. Nat. Photon 6, 829-833 (2012).

23. Shan, B. \& Chang, Z. Dramatic extension of the high-order harmonic cutoff by using a long-wavelength driving field. Phys. Rev. A 65, 011804 (2001).

24. Guggenmos, A. et al. Aperiodic CrSc multilayer mirrors for attosecond water window pulses. Opt. Exp. 21, 21728-21740 (2013).

25. Geissler, M. et al. Light propagation in field-ionizing media: extreme nonlinear optics. Phys. Rev. Lett. 83, 2930-2933 (1999).

26. Ammosov, M. V., Delone, N. B. \& Krainov, V. P. Tunnel ionization of complex atoms and of atomic ions in an alternating electromagnetic field. Sov. Phys. JETP 64, 1191-1194 (1986) 
27. Lewenstein, M., Balcou, P., Ivanov, M. Y., L'Huillier, A. \& Corkum, P. B. Theory of high-harmonic generation by low-frequency laser fields. Phys. Rev. A 49, 2117-2132 (1994).

28. Ivanov, M. Y., Brabec, T. \& Burnett, N. Coulomb corrections and polarization effects in high-intensity high-harmonic emission. Phys. Rev. A 54, 742-745 (1996).

29. Gordon, A. \& Kärtner, F. X. Quantitative modeling of single atom high harmonic generation. Phys. Rev. Lett. 95, 223901 (2005).

30. Brabec, T. \& Krausz, F. Intense few-cycle laser fields: frontiers of nonlinear optics. Rev. Mod. Phys. 72, 545-591 (2000).

31. Tong, X. \& Lin, C. Empirical formula for static field ionization rates of atoms and molecules by lasers in the barrier-suppression regime. J. Phys. B At. Mol. Opt. Phys. 38, 2593-2600 (2005).

32. Le, A.-T., Lucchese, R., Tonzani, S., Morishita, T. \& Lin, C. Quantitative rescattering theory for high-order harmonic generation from molecules. Phys. Rev. A 80, 013401 (2009).

33. Haworth, C. A. et al. Half-cycle cutoffs in harmonic spectra and robust carrierenvelope phase retrieval. Nat. Phys. 3, 52-57 (2007).

34. Balcou, P., Salieres, P., L'Huillier, A. \& Lewenstein, M. Generalized phasematching conditions for high harmonics: the role of field-gradient forces. Phys. Rev. A 55, 3204-3210 (1997).

\section{Acknowledgements}

We acknowledge support from MINISTERIO DE ECONOMA Y COMPETITIVIDAD through Plan Nacional (FIS2011-30465-C02-01), the Catalan Agencia de Gestió D’Ajuts Universitaris i de Recerca (AGAUR) with SGR 2014-2016, Fundacio Cellex Barcelona, support from Fundação para a Ciência e a Tecnologia (grant SFRH/BD/69913/2010) and funding from LASERLAB-EUROPE, grant agreement 284464.

\section{Author contributions}

F.S., S.M.T. and S.L.C. acquired the experimental data. J.B. conceived the attosecond set-up. M.H., S.L.C. and J.B. designed the laser system. J.B. and F.S. wrote the manuscript.

\section{Additional information}

Supplementary Information accompanies this paper at http://www.nature.com/ naturecommunications

Competing financial interests: There are no competing financial interests.

Reprints and permission information is available online at http://npg.nature.com/ reprintsandpermissions/

How to cite this article: Silva, F. et al. Spatiotemporal isolation of attosecond soft X-ray pulses in the water window. Nat. Commun. 6:6611 doi: 10.1038/ncomms7611 (2015).

\section{(c) (1)}

This work is licensed under a Creative Commons Attribution 4.0 International License. The images or other third party material in this article are included in the article's Creative Commons license, unless indicated otherwise in the credit line; if the material is not included under the Creative Commons license, users will need to obtain permission from the license holder to reproduce the material. To view a copy of this license, visit http://creativecommons.org/licenses/by/4.0/ 